\title{
A Prognostic Three-Axis Coordination Model for Supply Chain Regulation Using Machine Learning Algorithm
}

\author{
Hariprasath Manoharan, ${ }^{1}$ Yuvaraja Teekaraman $\mathbb{D D}^{2},{ }^{2}$ Ramya Kuppusamy, ${ }^{3}$ \\ Arun Radhakrishnan (iD, ${ }^{4}$ and Mohamed Yaseen Jabarulla (iD ${ }^{5}$ \\ ${ }^{1}$ Department of Electronics and Communication Engineering, Panimalar Institute of Technology, Chennai 600123, India \\ ${ }^{2}$ Mobility, Logistics, and Automotive Technology Research Centre, Faculty of Engineering, Vrije Universiteit Brussel, \\ Brussel 1050, Belgium \\ ${ }^{3}$ Department of Electrical and Electronics Engineering, Sri Sairam College of Engineering, Bangalore City 562 106, India \\ ${ }^{4}$ Department of Electrical \& Computer Engineering, Jimma Institute of Technology, Jimma University, Ethiopia \\ ${ }^{5}$ School of Electrical Engineering and Computer Science, Gwangju Institute of Science and Technology, \\ Gwangju 61005, Republic of Korea
}

Correspondence should be addressed to Yuvaraja Teekaraman; yuvarajastr@ieee.org and Arun Radhakrishnan; arun.radhakriahnan@ju.edu.et

Received 23 September 2021; Revised 14 October 2021; Accepted 3 November 2021; Published 25 November 2021

Academic Editor: Punit Gupta

Copyright (C) 2021 Hariprasath Manoharan et al. This is an open access article distributed under the Creative Commons Attribution License, which permits unrestricted use, distribution, and reproduction in any medium, provided the original work is properly cited.

\begin{abstract}
In this article, data processing of a supply chain management system has been monitored using the Internet of Space (IoS) which can be able to create possessions for managing the business process. In modern circumstances, many business inventiveness are trading and exporting products on their possession, but in many cases, information on such manufactured products is not monitored in an effective manner. To overcome the abovementioned issue, a precise model of monitoring several distributed products in supply chain management has been introduced with high sustainability error reduction. The framed model in the management process has been integrated with the boosting algorithm, a type of machine learning algorithm where training dataset has been introduced appropriately. This variation in the incorporation of the boosting optimization process not only increases the efficiency of the proposed model but also attempts to prove the success strategy under five different scenarios, where after sequential tests IoS model delivers high improvement in the distribution process for an average percentile of $67 \%$ than the existing methods.
\end{abstract}

\section{Overview on Internet of Space (IoS) in Supply Chain Management}

In day-to-day existence, the contrivance of data transfer is varying, and more amount of storage space is needed for the management process. Even after integration, most of the online cloud storage applications can be able to provide low storage whereat next period revitalization is necessary, which is a high cost optimization development model. If all these storage capabilities are lesser, then the sustainability rate of the technological process is lesser at high error rate. If such low susceptible methods are introduced in the supply chain management process, then it becomes very difficult to detect the movement of goods as it is a distribution process. Even in the supply chain management process, the distributed process can be monitored using the image-based technique, and they can be collected at different locations. But the major substance in the supply chain management process will be a new resource, which is framed in a natal mode at low storage capability.

In the exploration of guileless, it has been deliberated that IoS can be able to monitor the distribution of goods using existing antennas without high installation cost. Furthermore, IoS is used for analyzing significant data where lasers are pointed in free space, thus providing good communication in the management process. Even IoT can 
be used for the implementation of complex processes in supply chain applications. But low cost of implantation is not guaranteed, and error will be much higher, which leads to inadequate susceptible process. To avoid such inadequate process, old infrastructure can be converted to new detecting and data transfer techniques using IoS. Moreover, this IoS can be used as a backbone of any intelligent system as sudden propagations can be stopped at short period of time. Therefore, for a faultless distribution monitoring system, IoS has been integrated which even provides high-quality communication signals at remote locations shown in Figure 1.

1.1. Correlated Applications. In this section, all interconnected works on Internet of Space (IoS) applications have been discussed, which provides central ideas for the formation of the proposed work. However, the application of IoS has been updated from the Internet of Things (IoT), which provides high standard solutions to all problems addressed in the supply chain management process [1]. For providing solutions to an IoT process, big data analysis has been made, and solutions are based on the simulation model with a high variety of modifications with a new mathematical setup model. The solutions of the supply chain management monitoring process have been well defined in this collaborative model as high efficiency is observed with the minimization of risk in supply chain disturbance. By using the basic mathematical model, big data analysis has been further developed for analyzing the behavior of every consumer [2]. This type of model integrates regression analysis with the high forecast in demand, and at the same time, it minimizes the cost of installation. Also, a metaheuristic analysis on big data has been made using different algorithms which opens the path for innovative investigation in the areas of the supply chain monitoring process. Furthermore, the integration process is expanded by analyzing the approach of the data transfer technique which is referred to as the sustainable processing capability [3]. In such viable conditions, the parametric conditions such as the sustainability of data transfer to a receiver should be checked in a way that the integrated method should solve long-term problems that exists in real-life situation. The central system that provides support for the viable supply chain management process can be further classified with different adaptive mechanisms that ensemble in the area of high technological features.

Since an adaptive mechanism provides efficient solutions, it is also integrated for analyzing the effect of the smart grid process [4], where the nonlinear programming model is encoded for the optimization process. Even though the process aims to provide quick solutions to all prosumers, only implementation cost is observed with the distribution process. Moreover, this type of distribution is assimilated with the neural network which provides a solution to hidden grid terminals on a large scale, and this type of network is reliable to a greater extent. Another application that exists in the field of IoS is monitoring the emission of carbon-dioxide that is present in all industrial processes with central data distribution [5]. In this type of monitoring, authors have suggested an effectual model by considering multiple objectives, which reduces the effect of greenhouse gas, but there is no vital indication on the implementation cost. Conversely, case studies have been conducted in the Pareto frontier for defining the best model that is suitable in the real-time implementation process. Additionally, a review in the area of IoS is explored in analyzing the set of scales for Industry 4.0 where a diverse variable application has been enhanced [6]. While analyzing the each set of scales, the visibility level should be checked in a sensible manner, where data should be interrupted correctly. The abovementioned approaches differ from existing [1-5] methods in the absence of RFID tags which is a cost-saving process in the optimum method of creation of tags.

The importance of IoT has been further deliberated by considering 800 different journals which are developed as bibliometric analyses [7]. Most of the mechanisms implement the process of co-concurrence procedure which is specifically designed for industries and retail technology. By comparing different conventional procedures in the supply chain management process, more number of productions can be detected using IoS. One major entity that needs to be considered in IoS design is low-power networks [8] where, in wireless networks with low power, more amount of data should be transmitted at a short period of time. For every time period, the number of transmitted data will be updated as the process carried out using IoT by following the set of designed protocols. The procedure implemented in the lowpower wireless networks uses the Global Positioning System (GPS) as an enhancement module for monitoring the goods in the management process. But after comprehending that GPS can be replaced with advanced systems, a review on machine learning technique [9] which provides high-end automation for the supply chain management system has been deliberated. By using machine learning algorithms, all monitored data will be updated in the central server; therefore, all users can open the server with appropriate encoded keys.

Still apprising instruments are required for providing smart solutions in the supply chain management process; hence, high-end instruments are designed, which can be alleged as a smart model for the monitoring process [10]. Even if high amount of data is present in this kind of smart model, all information on trailing goods is provided. A separate numerical analysis has been carried out for establishing a coordination model that forms a new design strategy for future enhancing techniques [11], where single and multiple objective cases are considered. Subsequently, foundations on different algorithms are analyzed to model all networks, thus calculating the aggregate percentage of probable decisions that are observed in real time [12]. As a final point, it is essential to find the tractability of the supply chain management process to be implemented in real time; thus, two different types of flexibility are designed such as organization and product $[13,14]$. These two types enable the uses of the cost that provides an increase in the globalization of industries with optimal investments in IoS, thereby ensuring efficient worldwide operations. 


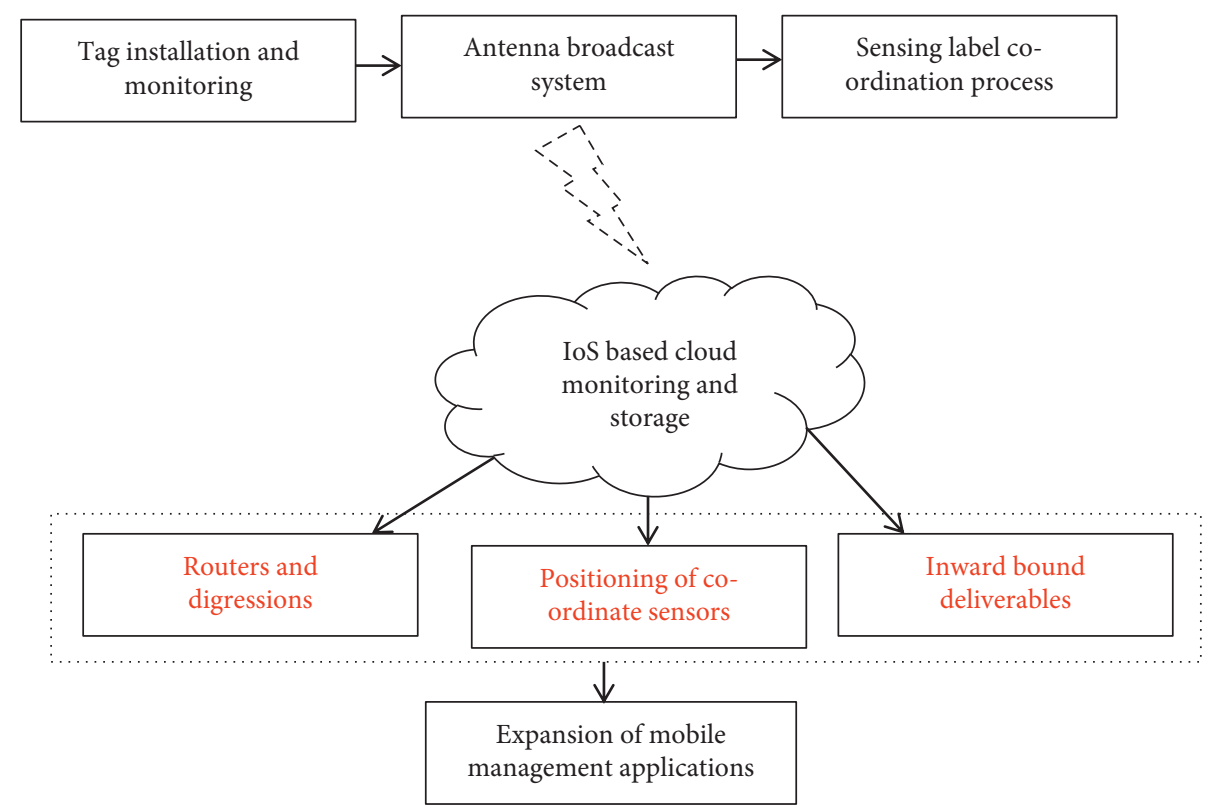

Figure 1: Schematic depiction of the proposed model using IoS.

1.2. Research Gap. Most of the existing methods describe only the impact of big data analysis using IoT for the supply chain management process by monitoring the changes in the distribution process. However, it has been witnessed that most of the methods are only experimental case studies, and even if implemented in real time, the distribution process cannot be monitored with changes at implemented locations. Even for storage capacity, a cloud-based monitoring system is not assimilated, which provides an abundant drawback. Moreover, it has been identified that metaheuristic examination has been carried out with high-efficient algorithms, but due to the lack of the distribution process, very low efficiency is delivered.

1.3. Proposed Methodology. Therefore, to overcome the abovementioned gaps and problems, a research problem has been formulated using a new technique which is termed as "IoS". The major motive of implementing IoS is that there is no prerequisite of the external cloud storage system, and all distribution processes can be easily monitored using the three-axis coordinate system. This diversity of changes is motivated from settlement-based systems that exploit wireless technology, and even it can be extended for sixth-generation systems. If a threeaxis coordinate system is framed, then the supply chain management process can be monitored without any involvement in the human recognition activity, and goods of all individual people can be examined at remote locations. Since synchronization is essential, an optimization data transfer approach which is termed as "boosting algorithm" is included, and efficiency is tested offline using MATLAB.

1.4. Objectives. The new flanged examination strategy on the application of the supply chain management process to analyze the movement of the distribution process will focus primarily on the following three major objectives: (i) To implement an IoS-based three-axis coordinate system in all industrial applications for monitoring the distribution process

(ii) To formulate a control strategy for detecting the association of goods with the secured data transfer process

(iii) To integrate boosting algorithm with the three-axis system for detecting the sustainability and pseudorange of the optimization process

\section{Mathematical Model of IoS for the Supply Chain Management}

This section describes the mathematical model of the Internet of Space for monitoring the supply chain management process, where, in the first segment, fundamental equations that are related to IoS are formulated using a defined pseudo range model where the satellite images will be monitored periodically for 30-second interval with a common reference clock. This type of measurement model can be framed as given in the following equation:

$$
\vartheta_{i}=\sum_{i=1}^{n}\left(R_{\mathrm{in}}-R_{s}\right) * \text { speed of light, }
$$

where $R_{\text {in }}$ and $R_{s}$ denote the interpretation of reading and receiver clocks, respectively.

Equation (1) indicates that the difference between two different clocks should be maintained within the pseudorange limits, and it should be multiplied with the speed of light which is equal to $299792458 \mathrm{~m} / \mathrm{s}$. However, equation (1) can be simplified using three different coordinate systems with unknown positions as modeled in the following equation: 


$$
\vartheta_{i}\left(R_{\mathrm{in}}, R_{s}\right)=\sum_{i, j, k=1}^{n} \sqrt{\left(i^{s}\left(\tau^{s}\right)-i(\tau)-j^{s}\left(\tau^{s}\right)-j(\tau)-k^{s}\left(\tau^{s}\right)-k(\tau)\right)}
$$

$$
\rho(i)=\frac{1}{2} \int_{0}^{\tau} e^{2} \mathrm{~d} e
$$

where $i^{s}, j^{s}$, and $k^{s}$ denote the corresponding satellite positions, $i, j$, and $k$ represent the receiver position that moves with respect to satellite systems, and $\tau^{\mathcal{s}}$ and $\tau$ denote the bias clock and reference clock that are changed in a periodic manner.

Since there is a necessity to determine linear equations, actual observations of different time intervals are modeled in the mathematical form and are given in the following equation:

$$
O_{i}=\vartheta_{i}\left(R_{\text {in }}, R_{s}\right)+n_{i}
$$

where $n_{i}$ denotes the amount of noise that is present in monitoring IoS system.

Equation (3) denotes that, original observations should be present with the low noise correlation capability where the positioning problem is solved with the exact linear observation model. In real time, it is not guaranteed that equation (3) will provide exact results; therefore, to reduce the error, the covariance matrix will be modeled as given in the following equation:

$$
E_{i}=\left(\begin{array}{ccc}
\sigma^{2} & \cdots & 1 \\
\vdots & \ddots & \vdots \\
1 & \cdots & \sigma
\end{array}\right) .
$$

Since in IoS a rotation clock is implemented, it is necessary to use a rotational matrix by considering latitude and longitude constraints with the local coordinate system as given in the following equation:

$$
\Delta r_{i}=R E_{i} \Delta_{i j k}
$$

where $R E_{i}$ denotes the rotation matrix that is varied with respect to equation (3). $\Delta_{i j k}$ represents the normalized error of all three coordinate systems.

Once the error matrix is found in the subsequent segment, the process of control operation should be incorporated that follows an integral technique with a dynamic system performance improvement. The control formulation for a dynamic supply chain management system can be framed as

$$
C(i)=\frac{1}{2} \int_{-\tau}^{+\tau} \in(i)+\epsilon(j)+\epsilon(k)
$$

where $\epsilon(i, j, k)$ denotes the corresponding elements in all three reference points.

Equation (6) indicates that integrative elements in all corresponding points should be reduced to half for monitoring the supply chain management process. In equation (6), the input needs to be entered, and the integral value of the management system should be within each state limits. Another important parameter for limiting the control process is the energy consumption that is monitored during supply chain management system, and it can be mathematically given in the integral form as where $e$ denotes the total energy consumed by each node.

The major reason for incorporating IoS in the supply chain management system is to check the level of variation in the capacity and rate of production involved in the process. The aforementioned parameters can be modeled using the following equation:

$$
\mu_{i} \frac{\partial I_{i}}{\partial \mu}=-\varphi_{i} \frac{\partial I_{i}}{\partial \varphi}
$$

where $\mu$ and $\varphi$ denote the corresponding capacity and production rate.

In the proposed method, IoS is incorporated for a product management where the cost of implementation depends on the changing capacity rate, and it can be calculated using the following equation:

$$
\operatorname{cost}(i)=\sum_{i=1}^{n} \frac{g(i j k)}{\varphi_{i}} \forall \operatorname{IoS},
$$

where $g(i j k)$ denotes the average number of goods that are measured at three reference points using IoS.

Equations (1)-(9) represent all basic formulations for the integration of IoS with the supply chain management application process. All abovementioned equations are formulated based on a control technique mechanism, thus providing global solutions in terms of production rate and capacity. Thus, the objective function can be framed using equations (1)-(9) as

$$
\operatorname{obj}(i)=\min \sum_{i=1}^{n} \rho(i) E_{i} C(i)
$$

The above equation indicates the minimization problem where a triobjective case study has been formed using minimized values of error, controller, and energy values. If all the parameters in triobjective functions are minimized, then the three-axis coordinate system can be implemented in real time with a great advantage of outpost-based images. Moreover, in equation (10), cost terms can also be added for the minimization process, which is much needed for mortality.

\section{Optimization Algorithm}

In this section, a precise optimization algorithm for managing the supply chain process in industries has been selected where all feature predictions can be made much easier using the boosting algorithm. This type of boosting algorithm is a type of machine learning algorithm where all estimated values in different regions can be integrated in a suitable manner. For any given input, the CatBoost algorithm [15-17] provides an accurate output by solving a different set of features where high loss can be controlled. 
Also, real-world application problems of the machine learning algorithm have been conferred for enhancing the intelligent process [16]. More quantities of data have been grouped where the data-driven approach for effective solutions has been secured for all industry authorities. With this review process, the secured mechanism is extended for detecting heart disease with big data on medical applications [17]. Even early detection is possible with the continuous observation of transience rate in humans, and the same approach can be extended for different applications which include supply chain management process. Since in the supply chain management process data are provided in different categories, this algorithm supports different category data such as text and numerical. In addition, fraud data can be detected, and it will be released in the next loop category; thus, the time of implementation is reduced. Also, training results in this type of boosting algorithm will be much higher using different command-line interfaces. In the starting segment of gradient boosting, constant values must be provided which provides high step implementation for further stages because 80 percent of the training set should be divided and 20 percent of the dataset should be provided initially. The process of initiating constant values in stage 1 can be framed as follows:

$$
\theta(i)=\sum_{i=1}^{n} \pi_{i}, \quad \forall i \exists n,
$$

where $\pi_{i}$ indicates the constant indicator function for all values that exist in all different stages between $i$ and $n$.

If equation (11) is integrated, then binary values should be indicated that provides the training data information for the boosting process. This can be framed using the following equation:

$$
\pi_{i}= \begin{cases}1, & \text { if } i \in n, \\ 0, & \text { otherwise. }\end{cases}
$$

If the condition in (12) is gratified, then approximation can be made with reduction of losses in overall network. Furthermore, to simplify the equations, an approximate function should be derived by combining equation (11) as

$$
Z_{i}=\sum_{i=1}^{n} \pi^{t}
$$

where $\pi^{t}$ indicates the sum of different approximations in corresponding time intervals.

Equation (13) provides valuable information on periodic time changes that are provided at different time periods. Since more number of datasets are represented, it is necessary to consider Time Division Multiple Access (TDMA) in this type of boosting algorithm. Furthermore, the update for every dataset can be represented using the following equation:

$$
|\widehat{A}(i)|=\frac{\min _{i} e_{i}^{t}}{e_{i}^{t-1}-e_{i}^{t+1}},
$$

where $e^{t}, e^{t-1}$, and $e^{t+1}$ represent the current, preceding, and next time samples for exchanging periodic information.
Once the dataset has been updated, this algorithm has to be integrated with the pseudorange model using the residual function as follows:

$$
\vartheta_{i}=\frac{\partial\left(R_{\mathrm{in}}, Z_{i}\right)}{\partial\left(R_{s}\right)} .
$$

The above equation indicates that the partial derivative of the reading clock and time period of each sample should be integrated for all corresponding pseudofunctions that are provided in three different coordinate systems. The step-bystep implementation of the CatBoost algorithm is provided in Figure 2, where it starts with the initialization of constant set values and the output will be based on exact prediction.

\section{Results and Discussions}

In this section, all formulated problems will be implemented with the projected algorithm, which in turn provides valuable solution for all monitored parametric values. This process is usually processed with the communication module which is termed as "transceiver," one important block for dispensation of hardware components. Since IoS is involved for monitoring all manufacturing equipment in the supply chain management process, an efficient hardware platform with a satellite-based system is needed for calculating the values with conventional objective functions. Furthermore, in this mechanism, there is no requirement for using different codes that are used as a reference purpose for identification. The abovementioned process is carried out to avoid misperception in all cases and the training set is initialized for detecting five different scenarios.

Scenario 1: control formation with error reduction

Scenario 2: limpidity on periodic intervals

Scenario 3: time of scale sustainability

Scenario 4: intensification of data security

Scenario 5: cost of implementation

Scenario 6: estimation on competence utility

For all five different scenarios, monitoring is carried out in both online and offline analyses, where, to understand the results in real time, the grids are plotted using MATLAB. Moreover, in MATLAB, all calculated component values are combined and only graphs are plotted, but all outcomes are observed in real time at periodic times in manufacturing industries. In the residual section, a detailed explanation about different scenarios is delivered.

4.1. Scenario 1. In this scenario, satellite systems are installed, and they are varied at different frequencies for the establishment of control information. If control information is established at an initial stage, then errors can be reduced in phase 1; else, errors will transpire through all phases under different paths, and as a result, due to high error values, the observed parametric values will rise above the threshold limit. Therefore, the control information is designed for all three coordinate axes using equations (1) and (2), where the reliability of the communication system is tested at two 


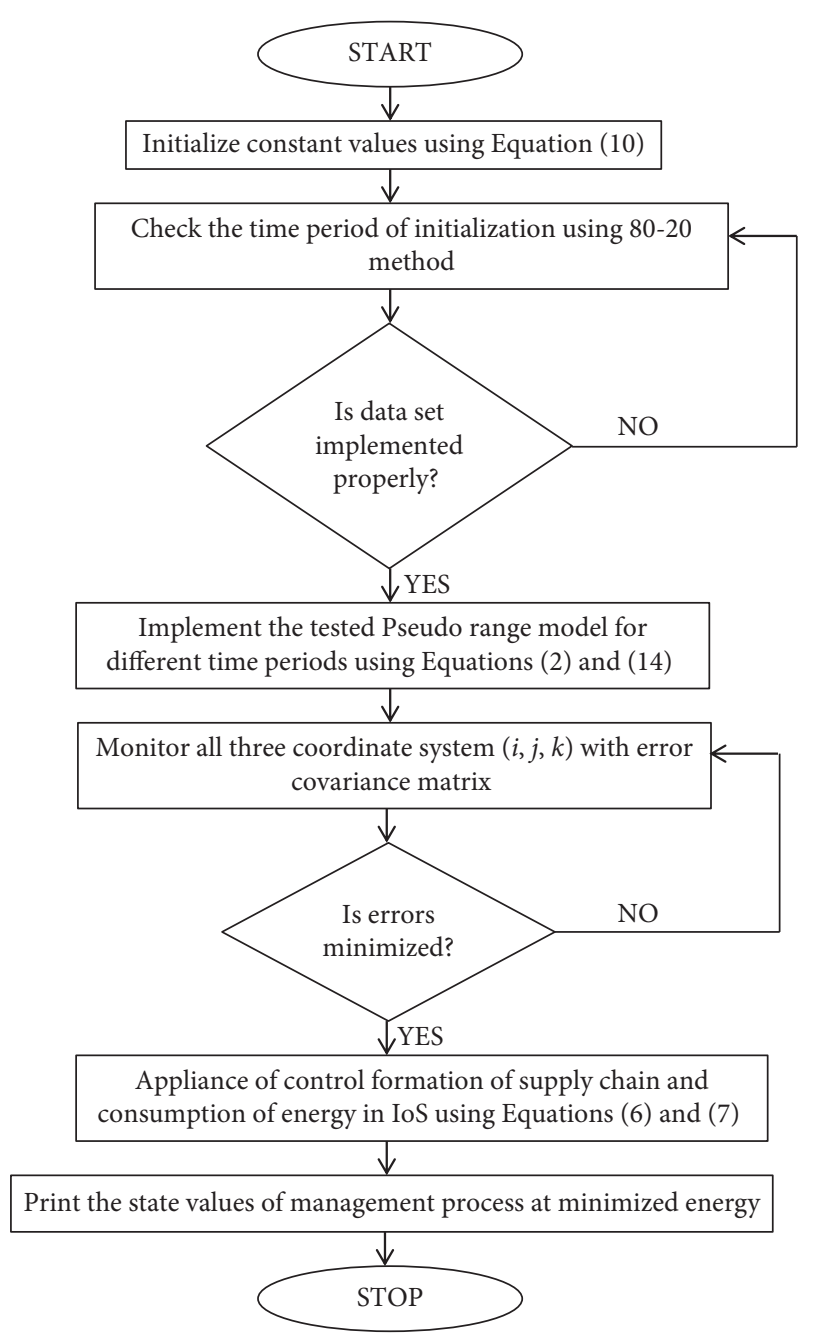

Figure 2: IoS for the supply chain management system.

different levels such as bit- and packet-level systems. In these two levels, if any failure is observed in equipped instruments, then a request will be directed to the central fault-tolerant system to delineate the probable limits. Thus, after observing the error values for different ranges of frequencies, Figure 3 is plotted using decibel values.

From Figure 3, it can be observed that a high degree of protection is assured for the proposed method when compared to existing methods [1]. To observe the parametric error values, frequency ranges are varied from 10 to 10000 Radians per second, and for each variation, error values are observed between -7 and -86 decibels for both proposed and existing methods. From offline analysis, it is much clear that an indicated marker for frequency of 1000 Radians per second provides an abundant difference in the error values of the existing method, as the proposed method delivers only low error values which is equal to -18 decibels. Since a high degree of accuracy is assured, the proposed method with the boosting algorithm can be integrated in real time with efficient control formation techniques.

4.2. Scenario 2. If error values are reduced in phase 1 , then transparency values for periodic times are calculated since the values are observed directly from the universe with three

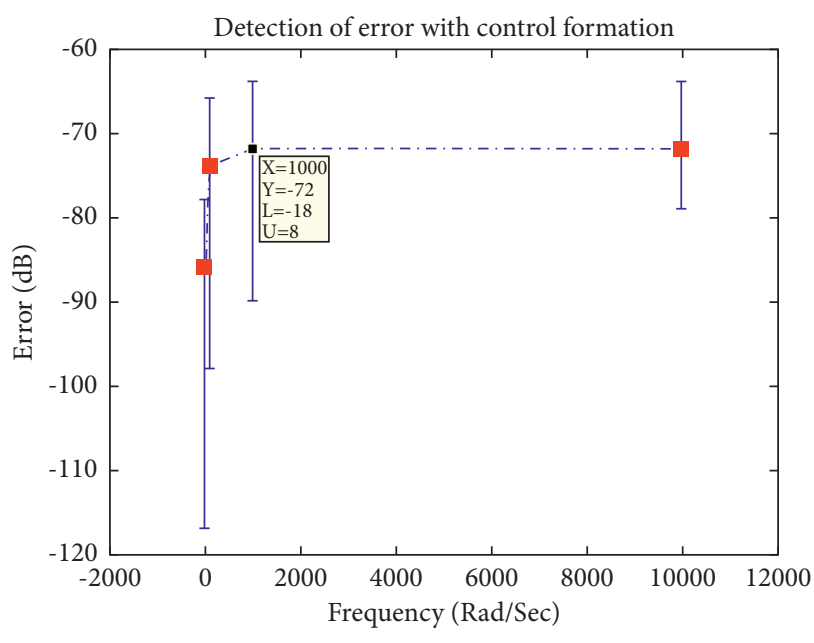

FIGURE 3: Observed error values for variation in frequency.

different axes. This scenario is considered primarily for monitoring all public organizations as the number of instruments is higher, and they are positioned using mobility nodes. Since the nodes are moving at different scales, transparency will be varied and a high amount of percentage values determines the efficient method. In case if the demand for different utensils rises, then a communication tool can be installed at an isolated subdivision to provide solutions for all raising issues with different constraints. For providing comprehensible results, the periodic time values are plotted in Figure 4.

From Figure 4, it can be perceived that, for different time periods, the percentage of transparency is calculated at a growing self-sufficiency mode. For monitoring the proposed mechanism, the time period is considered between 60 and 360 seconds, and it is found that the percentage of transparency is maintained at a constant rate when the proposed method is integrated with the boosting algorithm as the training dataset is implemented in a precise manner. If the training set is varied, then the transparency level will also vary, but in case if training dataset is not included, then error values will rise, and as a result, it will affect the transparency values. Furthermore, Figure 4 provides a clear indication that the transparency value of the existing method [1] is maintained only at 65 to 75 percentage whereas, for the proposed method, the transparency values are conserved at 92 to 96 percentage, which is much higher than that of the existing methods.

4.3. Scenario 3. This scenario evaluates the amount of maintainable rate that can be handled by IoS modules. Since the lifetime of the proposed method should be at least 10 years, it is necessary to arrange for an appropriate power supply subsystem. The major need for this power supply module is that the data-centric process will work efficiently when the power supply is designed in a precise manner. Also, sustainability can be evaluated based on different time periods, but in the first stage battery, life time should be improved for sustainable operation. Furthermore, the 


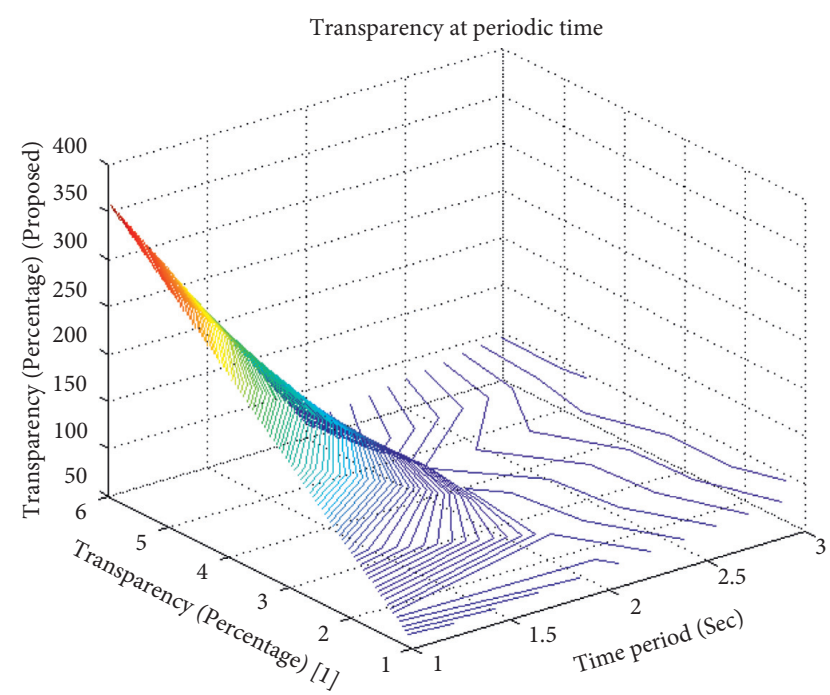

Figure 4: Percentage of transparency at periodic time interval.

central station should be designed using both transmitter and receiver modules that will be integrated using sufficient constraints. In the proposed method, three-axis coordinate systems are provided; therefore, a high sustainability rate should be observed which prevents the failure of the datacentric process. The observed sustainability rate is plotted in Figure 5.

From Figure 5, it can be detected that sustainability which is expressed in Kilo Watts per hour is determined in a varying rate using different time scales. For the small-time period monitoring, the time scales are taken between 60 and 360 seconds, and for each period, the rate of sustainability is maintained. Moreover, it is sensible that if the sustainability rate is maintained within $1 \mathrm{Kw} / \mathrm{h}$, then the efficient operation of IoS is assured. This is possible in the proposed method because for a periodic time, the IoS is sustainable at $0.8 \mathrm{Kw} / \mathrm{h}$, but with the same time period, the existing method [1] is sustainable at $4.3 \mathrm{Kw} / \mathrm{h}$. This proves that the proposed method can able to sustain at low power and at low frequency.

4.4. Scenario 4. The foremost constraint for any IoS model is to check the percentage of data that will be secured during the data transfer stage. The security of data is calculated based on the average percentage received from all coordinate axes; hence, if more amount of encryption keys is added, the data can be secured further. But the main challenging task in this type of application is due to the implementation of the wireless medium, and low security measures are provided for all fundamental operations. However, in the proposed method, since directional antennas are used, signals will be transferred in all directions; therefore, high security threats are present. Thus, the percentage of data security is measured and is plotted in Figure 6.

Figure 6 provides sufficient solutions for the average percentage of data that is integrated with the boosting algorithm. In the proposed method, the average data is considered between 2000 and 10000 meters, where for each

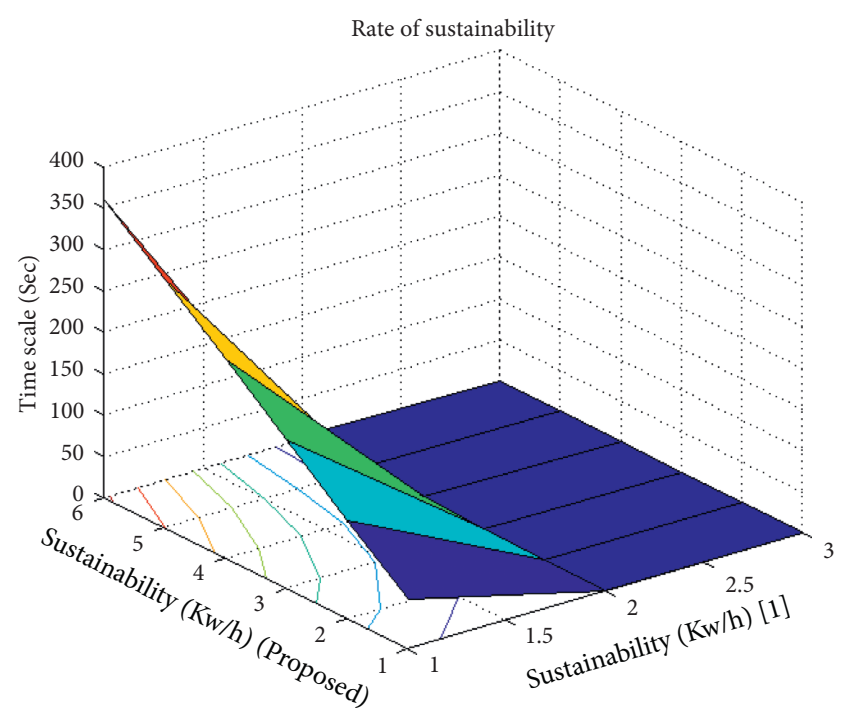

Figure 5: Rate of sustainability at periodic time scale.

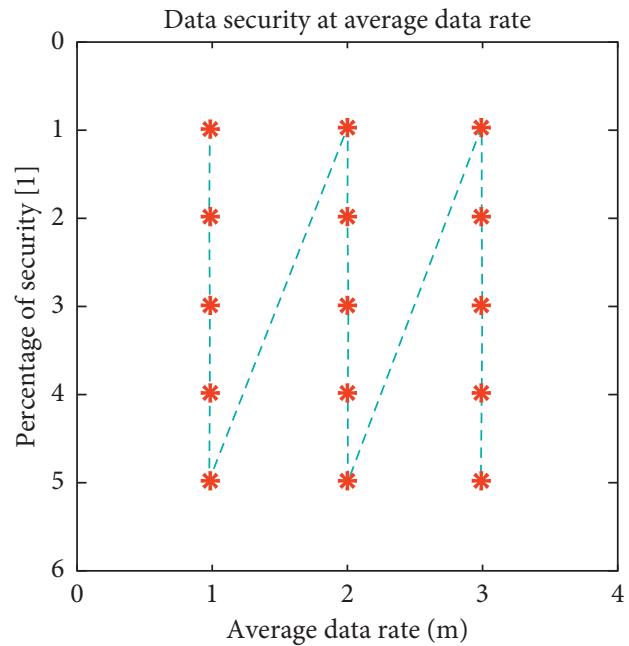

FIgURE 6: Security measures at average data rate.

meter, data security at all three axes is calculated. In case if the average data rate is considered as 6000 meters, then the security of data for the existing method will be 68 percentage, whereas, for the proposed method, 85 percentage of data is secured. Moreover, for all average data rates, the proposed method secures the data for more than 80 percentage, thus resulting in achieving efficient safeguard of data.

4.5. Scenario 5. In the proposed method using IoS, the cost of implementation will not be much similar to the basic IoT mechanism as three coordinate axes need to be designed with satellite installation. Therefore, the cost of implementation will be higher when compared to IoT, but monitoring performance will be higher. Thus, the cost parameter for installation can be contented to some extent of available resources. In the proposed method, the cost of implementation is calculated based on the capacity rate of 


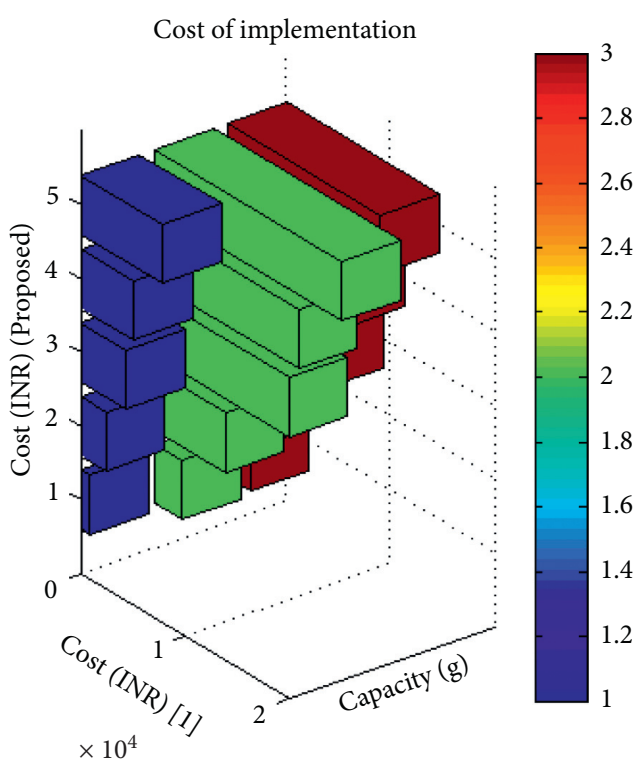

FIGURE 7: Implementation cost: proposed vs. existing.

available resources. The analyzed value for the implementation of the cost parameter is shown using a threedimensional bar graph in Figure 7.

Figure 7 indicates the installed capacity in grams which is varied from 800 to 7800 , and for these varying capacities, the cost of installed units is calculated. It is evident from the horizontal bar that the cost of installation of the more number of units in the proposed method is much lesser than that in the existing method. For example, if the capacity of installed units is 5000 grams, then the cost of installation is 13765 INR for the proposed method, whereas, with the same amount of capacity, the existing method implements a cost of 11378 INR which is one percentage higher than the existing methods. This indicates that the performance of the proposed method is higher at low cost of installation.

4.6. Scenario 6. In this scenario, the best fitness function is calculated by varying the number of variables, where depending on the weight of each integrated outposts, further the values will be maximized at the output. Every value of the fitness score is denoted for each variable; hence, a separate value is assigned, and an individual model is created using equation (10). In addition, for the modeled scenario, a timebased solution is provided which makes multiple tasks to be performed at the same time. Also, a three-axis coordinate system which specifies a Boolean representation is made with the help of historic data in the optimization problem. After integrating the projected objective with different case studies, the fitness function is evaluated in MATLAB and is deliberated in Figure 8.

From Figure 8, in the first stage, it can be seen that the number of generations is observed per second and a subsequent variable design is made. In the next stage, depending on the designed variables, fitness function is observed where in the proposed method values are found to be less than 0.5 which indicates a best fitness function is achieved at low

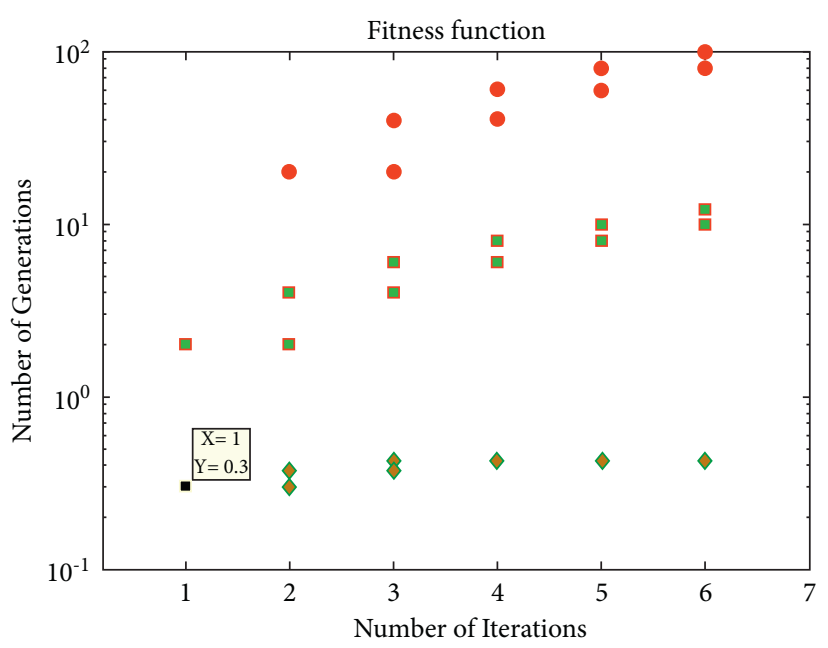

Figure 8: Effective fitness function of the proposed model.

generated values with 100 . Moreover, a unique model has been designed for achieving this fitness function, and an unrestricted mode is switched on at different dynamic positions. At the final stage, the generated process is encoded with different values where it will be converted from outpost values to normally generated values.

\section{Conclusions}

Based on the awareness of supply chain management in the distributed system, an IoS procedure for the system model has been introduced in this article. In the proposed method, a new measurable model for the supply chain management process has been deliberated where all imports and exports can be monitored with the help of a three-axis coordinate system. However, this type of system can be established, and they can be able to monitor the abovementioned management parameters since the method has been extended from IoT. But the major demand is controlling the error during such monitoring process in public administrations as a small error will cause the system to obscure. Therefore, exertions have been taken for reducing the error of measurement at periodic intervals, and the percentage of sustainability has been extended by installing the IoS route with the boosting algorithm. By comparing the proposed method with the existing one, the investigation has been extended with several new parameters, and in addition, the proposed method has not only stopped in the stage of monitoring. This proves that the projected technique is implemented not only for monitoring, but also to solve real-time problems, efforts have been taken, and after comparing five different scenarios with the existing method, the proposed method proves to be much efficient in terms of sustainability and error reduction.

\section{Data Availability}

The data used to support the findings of this study are available from the corresponding author upon request. 


\section{Conflicts of Interest}

The authors declare that they have no conflicts of interest.

\section{References}

[1] W. Jiang, "An intelligent supply chain information collaboration model based on Internet of Things and big data," IEEE Access, vol. 7, pp. 58324-58335, 2019.

[2] M. Seyedan and F. Mafakheri, "Predictive big data analytics for supply chain demand forecasting: methods, applications, and research opportunities," Journal of Big Data, vol. 7, no. 1, 2020.

[3] D. Ivanov, "Viable supply chain model: integrating agility, resilience and sustainability perspectives-lessons from and thinking beyond the COVID-19 pandemic," Annals of Operations Research, vol. 23, 2020.

[4] N. Saker and L. Kerbache, "Modeling smart grids using closed loop supply chain concepts," International Journal of Smart Grid and Clean Energy, vol. 15, pp. 8-14, 2014.

[5] F. Niakan, A. Baboli, V. Botta-Genoulaz, R. TavakkoliMoghaddam, and J. P. Camapgne, "A multi-objective mathematical model for green supply chain reorganization," IFAC Proceedings Volumes (IFAC-PapersOnline), vol. 46, 2013.

[6] S. Ahmed, T. Kalsoom, N. Ramzan et al., "Towards supply chain visibility using Internet of Things: a dyadic analysis review," Sensors, vol. 21, no. 12, pp. 1-24, 2021.

[7] A. Rejeb, S. Simske, K. Rejeb, H. Treiblmaier, and S. Zailani, "Internet of Things research in supply chain management and logistics: a bibliometric analysis," Internet of Things, vol. 12, Article ID 100318, 2020.

[8] S. Yuvaraj and M. Sangeetha, "Smart supply chain management using Internet of things(IoT) and low power wireless communication systems," in Proceedings of the 2016 IEEE International Conference on Wireless Communications, Signal Processing and Networking, WiSPNET 2016, pp. 555-558, Chennai, India, March 2016.

[9] D. Jhala, "Review on smart supply chain management using IoT and machine learning," International Journal of Innovative Research in Science, Engineering and Technology, vol. 42, 2019.

[10] H. Elmesmary and A. S. Gamal Abd El-nasser, "Smart solutions for logistics and supply chain management," International Journal of Recent Technology and Engineering, vol. 8, no. 4, pp. 2996-3001, 2019.

[11] Y. H. Lee, P. Golinska-Dawson, and J. Z. Wu, "Mathematical models for supply chain management," Mathematical Problems In Engineering, vol. 2016, Article ID 6167290, 4 pages, 2016.

[12] S. Y. Barykin, I. V. Kapustina, S. M. Sergeev, and V. K. Yadykin, "Algorithmic foundations of economic and mathematical modeling of network logistics processes," Journal of Open Innovation: Technology, Market, and Complexity, vol. 6, no. 4, pp. 1-16, 2020.

[13] G. M. G. Farok, "Mathematical modeling for measures of supply chain flexibility," Journal of Mechanical Engineering, vol. 45, no. 2, pp. 96-117, 2016.

[14] P. Pourhejazy and O. K. Kwon, "The new generation of operations research methods in supply chain optimization: a review," Sustainability, vol. 8, no. 10, 2016.

[15] W. Liu, K. Deng, X. Zhang et al., "A semi-supervised tricatboost method for driving style recognition," Symmetry, vol. 12, no. 3, pp. 1-18, 2020.
[16] I. H. Sarker, "Machine learning: algorithms, real-world applications and research directions," SN Computer Science, vol. 2, no. 3, pp. 1-21, 2021.

[17] B. H. C. C. Priya and K. Viswavardhan Reddy, "Performance analysis of machine learning algorithms for disease prediction," in Proceedings of the 2021 Grace Hopper Celebration India, Bangalore, India, March 2021. 